\section{El Sistema de Planificación del Perú y su Impacto en el Desarrollo Socio Económico}

\author{
The Planning System of Peru and its Impact on \\ Socio-Economic Development
}

\section{RESUMEN}

El presente artículo se orienta al desarrollo del sistema de planificación del Perú y su impacto en la evolución socio-económica con respecto a los factores del macro entorno internacional (globalización) y nacional (sectores que comprende a los recursos no renovables como los minerales y renovables (como la agro industria, textil, etc.) y sus factores políticos, económicos, sociales, regulatorios, culturales, turismo, medio ambiente, tecnológico (patentes y propiedad intelectual) etc. Y su micro entorno, como es el comportamiento del mercado nacional (oferta y demanda) con sus respectivos indicadores de desarrollo. En toda sociedad y particularmente en nuestro país el intangible más importante es el talento humano antes llamado recurso humano (competencias cognitivas, las habilidades blandas y afectivas, liderazgo, creatividad e innovación). Con mayor razón en los países emergentes como el nuestro, donde los gobiernos deben generar un clima de estabilidad política, social y económica de inclusión social, con una educación de calidad, apostando por el desarrollo de la innovación y creatividad, cero tolerancias a la inseguridad ciudadana y tener un rol promotor de la responsabilidad social y del emprendimiento, factores claves para el desarrollo del Perú. En un mundo globalizado, en la que las tecnologías de la información y comunicaciones se modernizan permanentemente, como sus herramientas internet, intranet, redes sociales, plataformas virtuales, programas informáticos, etc., generan la necesidad de que los sectores estén integrados en un sistema de información nacional. Todo esto será posible si se combate desde de todos los frentes la inmoralidad, que genera desconfianza y falta de credibilidad en sus autoridades.

Palabras claves: Sistema de Planificación; Globalización; Propiedad Intelectual y Responsabilidad Social.

\section{Luis Fernando Valeriano Ortiz}

lvalerianoo@unmsm.edu.pe

Universidad Nacional Mayor de San Marcos, Facultad de Ciencias Administrativas 


\begin{abstract}
This article focuses on the development of Peru's planning system and its impact on socio-economic developments with respect to the factors of the international (globalization) and national macro environment (sectors that include non-renewable resources such as minerals and renewable (such as agro industry, textile, etc.) and its political, economic, social, regulatory, cultural, tourism, environmental, technological factors (patents and intellectual property) etc. And its micro environment, such as the behavior of the national market (supply and demand) with their respective development indicators. In every society and particularly in our country the most important intangible is the human talent formerly called human resource (cognitive skills, soft and emotional skills, leadership, creativity and innovation). With greater reason in emerging countries like ours, where governments must generate a climate of political, social and economic stability of social inclusion, with a quality education, betting on the development of innovation and creativity, zero tolerances to insecurity citizen and have a promoting role of social responsibility and entrepreneurship, key factors for the development of Peru. In a globalized world, in which information and communications technologies are constantly modernized, such as their internet tools, intranet, social networks, virtual platforms, computer programs, etc., generate the need for sectors to be integrated into a system of national information. All this will be possible if immorality is fought from all fronts, which creates distrust and lack of credibility in its authorities.
\end{abstract}

Keywords: Planning; Globalization; Intellectual Property and Social Responsibility System.

\section{INTRODUCCIÓN}

El Perú es un país que, en las últimas cuatro décadas, viene manteniendo un desarrollo equilibrado y sostenido de sus factores macro económicos reflejados en sus indicadores más importantes como es el Producto Bruto Interno (PBI), que según fuentes de organismo multinacionales como es el Fondo Monetario Internacional que señala $2.6 \%$ de crecimiento del Perú en el 2019, porcentaje superior al promedio de los países de América Latina para el mismo periodo que es del $0.8 \%$. La crisis económica internacional generada por los Estados Unidos de Norteamérica y la China por aspectos arancelarios en sus exportaciones e importaciones de sus productos repercute en las economías mundiales y principalmente en los países exportadores de productos tradicionales (minerales: oro, cobre, plata y zinc) como es el caso del Perú. De igual forma, la crisis política interna que vive el país y con el enfrentamiento de los poderes del estado: ejecutivo y legislativo, ha desencadenado el cierre del congreso, ha generado una desaceleración de las inversiones nacionales y extranjeras en los sectores pro- ductivos, servicios y comerciales del país por la inseguridad jurídica.

Dentro de esta realidad la economía peruana se mantiene positiva, la ventaja competitiva del país son sus abundantes recursos naturales en la costa, sierra, selva y en el mar, la agro industria (exportaciones de espárragos, café, palta, quinua, cacao, arándanos, etc.) y el sector turismo (riqueza en el patrimonio cultural y arqueológico), que vienen generando divisas que son el respaldo del fortalecimiento y la sostenibilidad del desarrollo el Perú.

El Perú requiere desarrollar un Sistema de Planificación Estratégico a largo plazo con inclusión social con un modelo de economía de mercado, que tome en cuenta una visión de 30 años, que contemple una reingeniería de la clase política, fortaleciendo los partidos políticos, apostando por un sistema de gobierno digital en sus procedimientos y operaciones sectoriales, una educación de calidad en todos los niveles basados en el licenciamiento y acreditación, priorizar la investigación y desarrollo reflejadas en patentes y propiedad intelectual, cero tolerancia a la inseguridad ciudadana, identifi- 
cando las prioridades sectoriales productivas y de servicios de las 25 regiones del país para ser atendidas presupuestalmente. Potenciar la recaudación y fiscalización tributaria, sin exoneraciones principalmente a la gran empresa y universidades privadas. En el frente externo fortalecer los tratados de libre comercio suscritos por el Perú con países del mundo y formar parte de alianzas estratégicas con países de interés comercial. El Perú es un país que a lo largo de su historia ha sido mutilado geográficamente, esta situación no se debe repetir, tenemos que tener unas fuerzas armadas disuasivas que neutralicen cualquier intento de confrontación con países vecinos asignándoles los recursos presupuestales necesarios.

\section{OBJETIVOS DEL ENSAYO}

El objetivo del artículo es proponer un Sistema de Planificación Estratégico del Perú a largo plazo en función a los planes de desarrollo de los sectores productivos, sociales y económicos del país y su implicancia en los Planes Estratégicos Empresariales. De igual forma, contribuir al desarrollo socioeconómico del país conectado con los programas de responsabilidad social mediante un Sistema de Planificación integral.

\section{ARGUMENTACIÓN}

El Sistema de Planificación del país requiere tomar en cuenta de los factores e indicadores del macro y micro entorno nacional e internacional con sus correspondientes indicadores mensurables y cualitativos para fijar su temporalidad y desarrollo socio-económico.

\section{Análisis de Macro Entorno Nacional.}

\section{Factor Político.}

El riesgo país está relacionado con las inversiones nacionales y extranjeras que se ejecutan en el Perú en un periodo determinado, en la que se evalúa los impactos políticos, económicos, sociales, seguridad pública, etc. en la gestión gubernamental.

Según fuentes internacionales de entidades especializadas como el Banco de Inversión JP Morgan el riesgo país de Perú 2019 bajó cuatro puntos básicos y cerró en 0.91 puntos por- centuales y reportó el riesgo país más bajo de la región, a pesar de la crisis política que vive con el enfrentamiento de los poderes del estado.

\section{Factor Económico.}

El factor macro económico más importante es el PBI para un país, que se refleja en porcentajes, permitiendo conocer su evolución e involución económica, expresa el valor monetario de la producción de bienes y servicios de demanda final de un país durante un período determinado.

El Fondo Monetario Internacional (FMI) redujo la proyección de crecimiento de Perú para el 2019 de $3.5 \%$ a $2.6 \%$, según su informe de "Perspectivas Económicas Globales" y espera que la economía avance $3.6 \%$ el próximo año.

\section{Factor Social.}

Poblaciones Vulnerables.

El Perú ha reducido su porcentaje de población en situación de pobreza multidimensional de 20\% a 12.7\%, en el 2019 destacó el Programa de las Naciones Unidas para el Desarrollo como el Programa de Alimentación Escolar Qali Warma programa del Ministerio de Desarrollo e Inclusión Social (MIDIS) que brinda alimentación variada y nutritiva a niñas y niños de nivel de educación inicial y primaria en las escuelas públicas de todo el Perú, además del nivel secundario en las poblaciones indígenas de la Amazonía peruana.

\section{Sistema Educativo.}

PISA es un programa para la Evaluación Internacional de Estudiantes (PISA) de la Organización para la Cooperación y el Desarrollo Económico (OCDE) que se aplica cada 3 años y busca conocer en qué medida los estudiantes de 15 años son capaces de utilizar los conocimientos y habilidades de competencia lectora, competencia matemática y competencia científica. La prueba PISA se aplicó en Perú del 13 de agosto al 30 de septiembre de 2018.

Lamentablemente el Perú ocupa el penúltimo lugar, lo que demuestra el desinterés de los gobiernos por la educación peruana de la básica regular. 
Situación parecida sucede con la educación superior, no se figura en los rankings internacionales de universidades de calidad en la formación profesional dentro de las 500 primeras universidades. En ese sentido, la mayoría de las universidades peruanas están en proceso de licenciamiento y pocas están acreditas por la Superintendencia Nacional de Educación Superior Universitaria (SUNEDU).

El sector educación es estratégico para el desarrollo de país y debe ser comprendido en la planificación nacional como prioridad.

\section{Sistema de Salud.}

La tasa de mortalidad infantil se usa como un indicador del nivel de salud en el país. Asimismo, evalúa la mortalidad durante el primer año de vida. La tasa es el cociente entre el número de muertes y el total de nacidos vivos del mismo período, por mil. En el Perú, la tasa de mortalidad infantil 2018:

Total: 19 muertes/1.000 nacimientos

Hombres: 21,1 muertes/1.000 nacimiento

Mujeres: 16,7 muertes/1.000 nacimientos.

\section{Factor Ambiental.}

En la Planificación Nacional se hace necesario tomar en cuenta el valor del impacto ambiental fundamentalmente en:

- Disminución del suministro de agua.

- Calidad de aire.

- Biodiversidad y número de especies en peligro.

- Niveles de contaminante en sangre.

- Estudios epidemiológicos.

- Cantidad o calidad de recursos naturales.

- Reducción de ozono atmosférico.

En el Perú el impacto ambiental sobrepasa los límites de los estándares internacionales, afectando la salud y el ecosistema. El sistema de planificación debe comprender políticas de protección del medio ambiente en todos los sectores y de combatir la contaminación en el ámbito nacional.

\section{Factor Regulatorio.}

El sistema judicial del país requiere de una restructuración integral, que comprenda la revisión de los códigos penales, civiles, laborales, administrativos, tributarios, etc. con observancia a la Constitución Política del país. De igual forma se requiere de una evaluación y capacitación de los magistrados de las diversas instancias judiciales. Se le debe dotar de una infraestructura adecuada y tecnología informática para agilizar los procedimientos judiciales.

\section{Factor Tecnológico.}

Apostar por las tendencias tecnológicas de digitalización en la planificación nacional como los:

- Teléfonos Inteligentes: los smartphones continuarán integrándose a las vidas de las personas.

- Uso Intensivo de Redes Sociales: Facebook, Twitter, Instagram, YouTube, etc.

\section{Digitalización para el Servicio al Cliente.}

La Inteligencia Artificial: posibilidad de analizar la data y contar con un sistema de información informático.

\section{Fuentes de Energía Renovables.}

Las energías renovables son aquellas que se obtienen a partir de fuentes naturales inagotables como:

- Energía solar: Es aquella energía que es producida a través de los rayos del sol. por ejemplo, los satélites del espacio incorporan paneles solares para abastecerse energía.

- Energía Eólica: energía producida a través del viento.

- Energía Hidráulica: energía que es producida a través del agua.

- Energía Geotérmica: extracción de calor del interior de la Tierra.

- Energía Biomasa: se puede utilizar para cualquier tipo de residuos orgánicos, deposiciones, larvas a insectos, restos de ramas y hojas, etc. 
Fuentes de Energía no Renovables.

Estas son las principales fuentes de energía no renovables como:

- Energía de fuentes fósiles: Es la energía generada por el carbón, o el petróleo y derivados.

- Energía nuclear: Proporcionada por el uranio como su combustible principal.

\section{Análisis del Macro Entorno Internacional.}

\section{Factores Globales.}

Las crecientes tensiones comerciales y arancelarias entre Estados Unidos de Norteamérica y la China afectan las economías de los países del mundo y en forma muy especial en los países que exportamos minerales, que son productos que están sujetos a las alzas y bajas de la cotización de sus precios de acuerdo con el mercado de valores a nivel mundial afectando el futuro de las cadenas de valor mundiales. Esta situación coyuntural debe ser tomada en cuenta en la planificación nacional.

\section{Factor Tratados de Libre Comercio.}

El Perú en las últimas décadas viene suscribiendo varios tratados de libre comercio con países estratégicos como Estados Unidos de Norteamérica, países integrantes del mercado común europeo, países asiáticos, países latinoamericanos, etc. lo que permite exportar productos tradicionales como la plata, oro, cobre y zinc y no tradicionales aprovechando las políticas de la diversificación productiva como los espárragos, arándanos, café, cacao, quinua, etc. con cero aranceles, lo que significa mejorar nuestra balanza comercial e ingresos de divisas fortaleciendo la economía del país. Nuestro país siempre debe aplicar políticas de salvaguardias para defender aquellos productos y servicios de origen nacional que se verían afectados. La planificación nacional debe tomar en cuenta el impacto económico que genera los tratados de libre comercio en el desarrollo sectorial del país.

El presente artículo reflexivo va a dar origen al desarrollo de mi investigación denominada "La Planificación en el Perú y su Impacto en el Desarrollo Socio-Económico", a continuación, planteo el esquema que es materia del desarrollo de la investigación (Ver Figuras 1-4).

\section{CONCLUSIONES}

La Constitución Política del Perú establece en su Artículo 58 que:

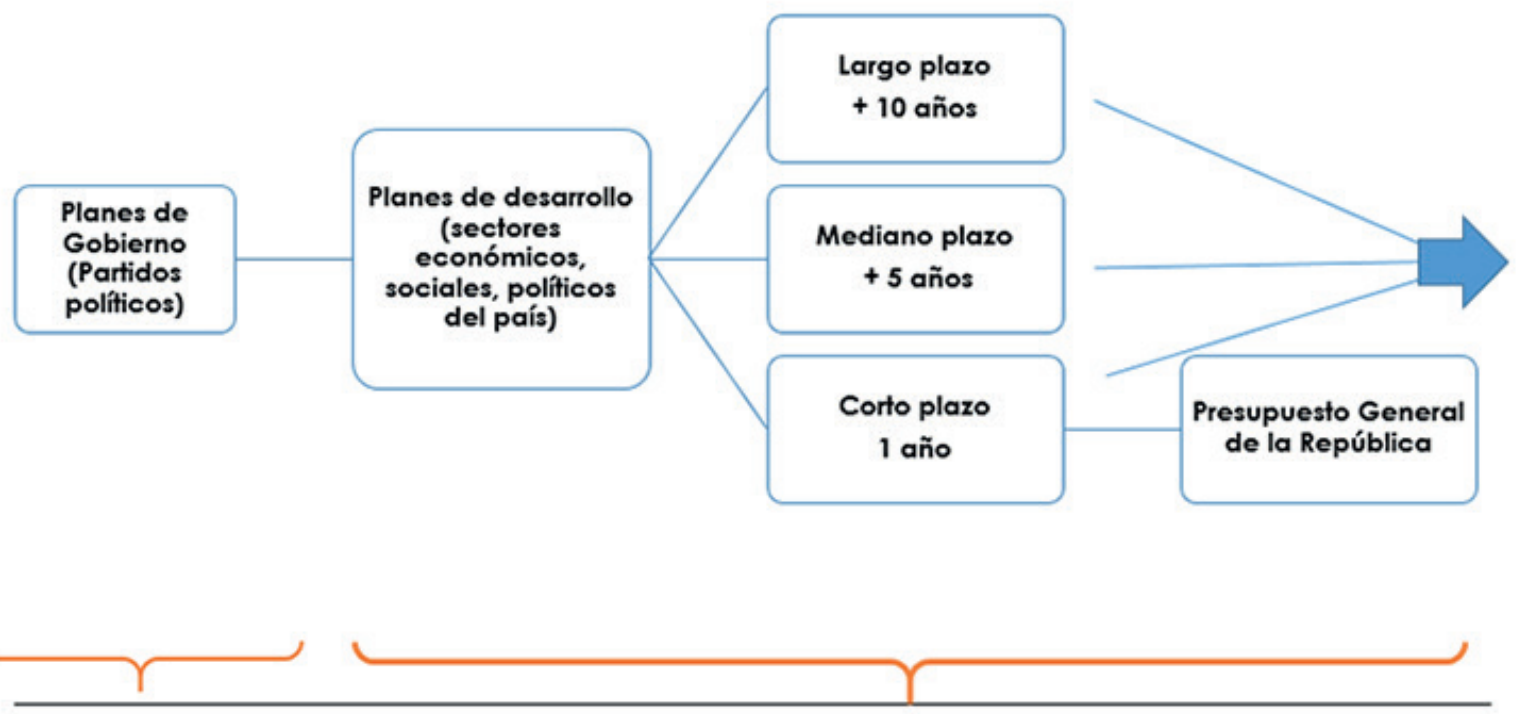

Proceso Electoral Sector Público Nacional

Figura 1. Sistema de Planificación del Perú y su Impacto en el Desarrollo Socio-Económico (Parte I). Elaboración propia 


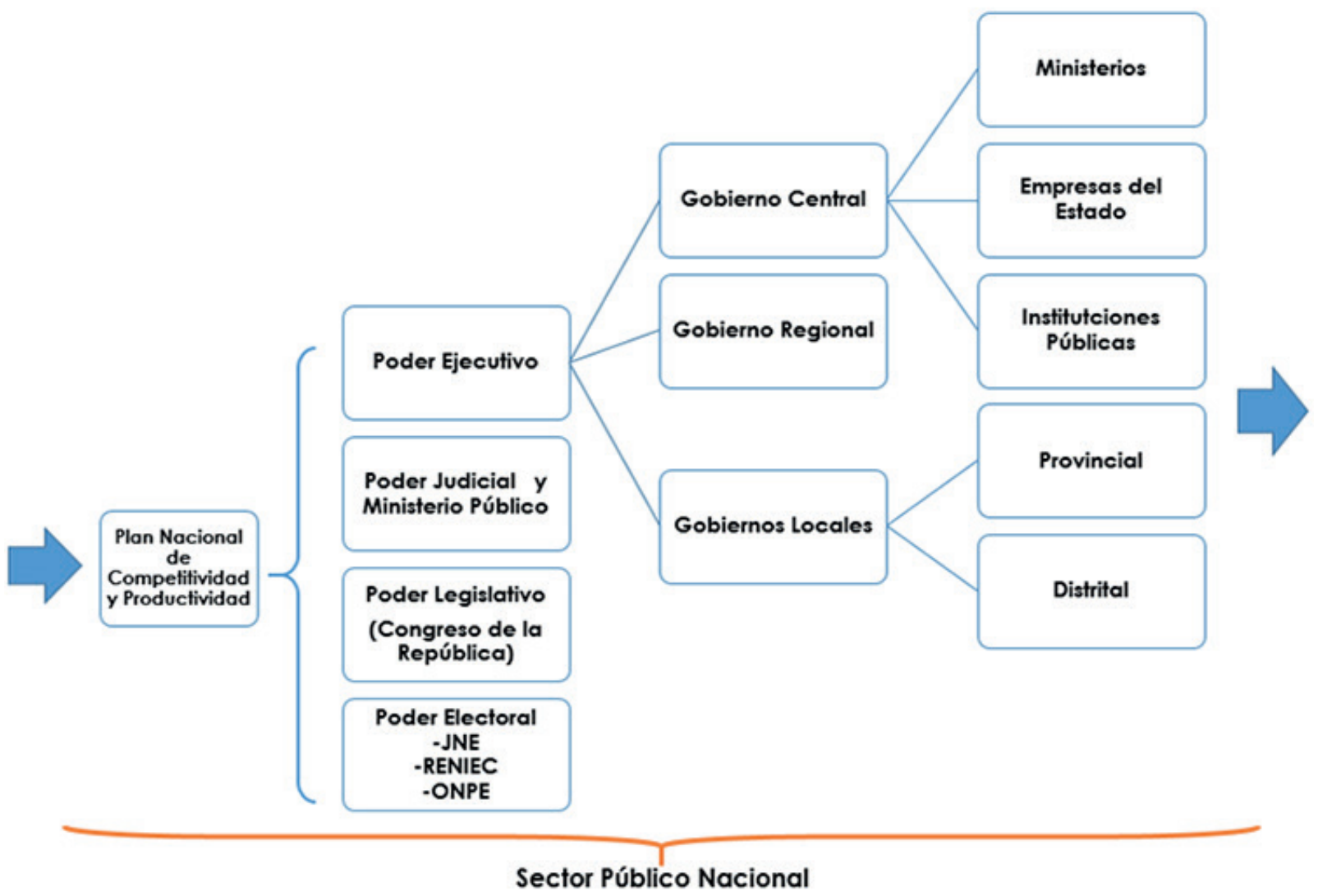

Figura 2. Sistema de Planificación del Perú y su Impacto en el Desarrollo Socio-Económico (Parte II). Elaboración propia
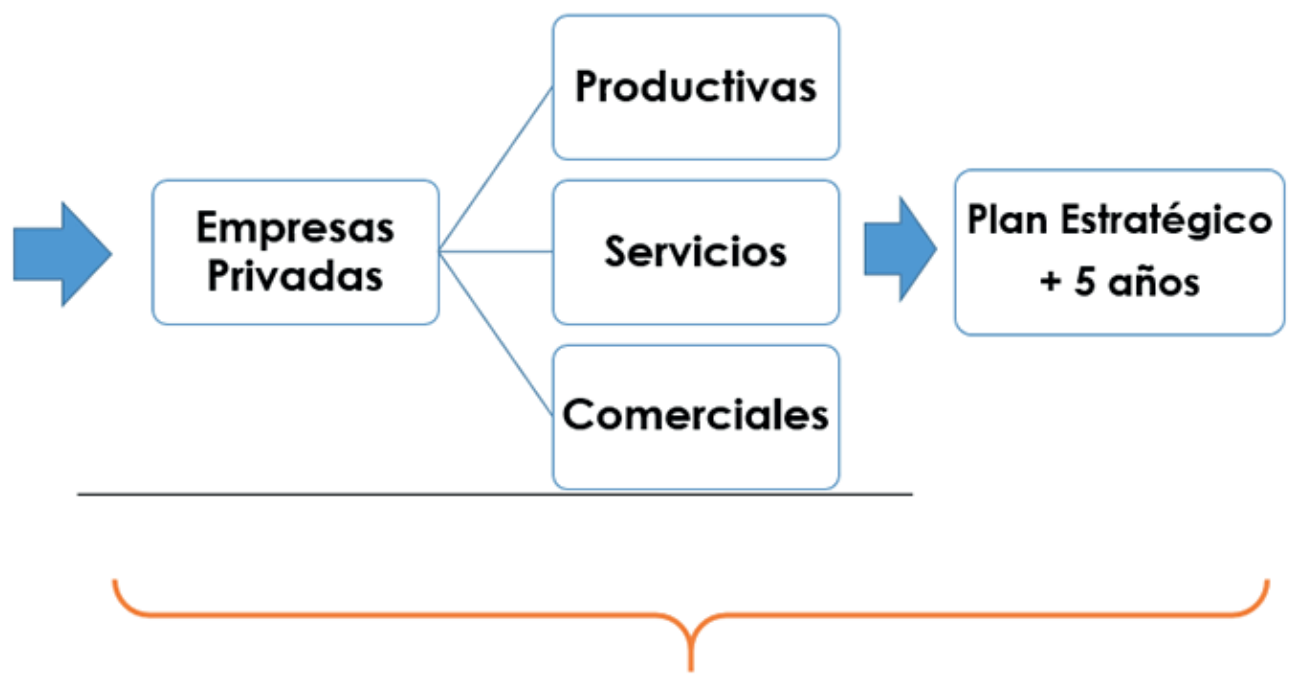

\section{Ámbito Empresarial}

Figura 3. Sistema de Planificación del Perú y su Impacto en el Desarrollo Socio-Económico (Parte III).

Elaboración propia 


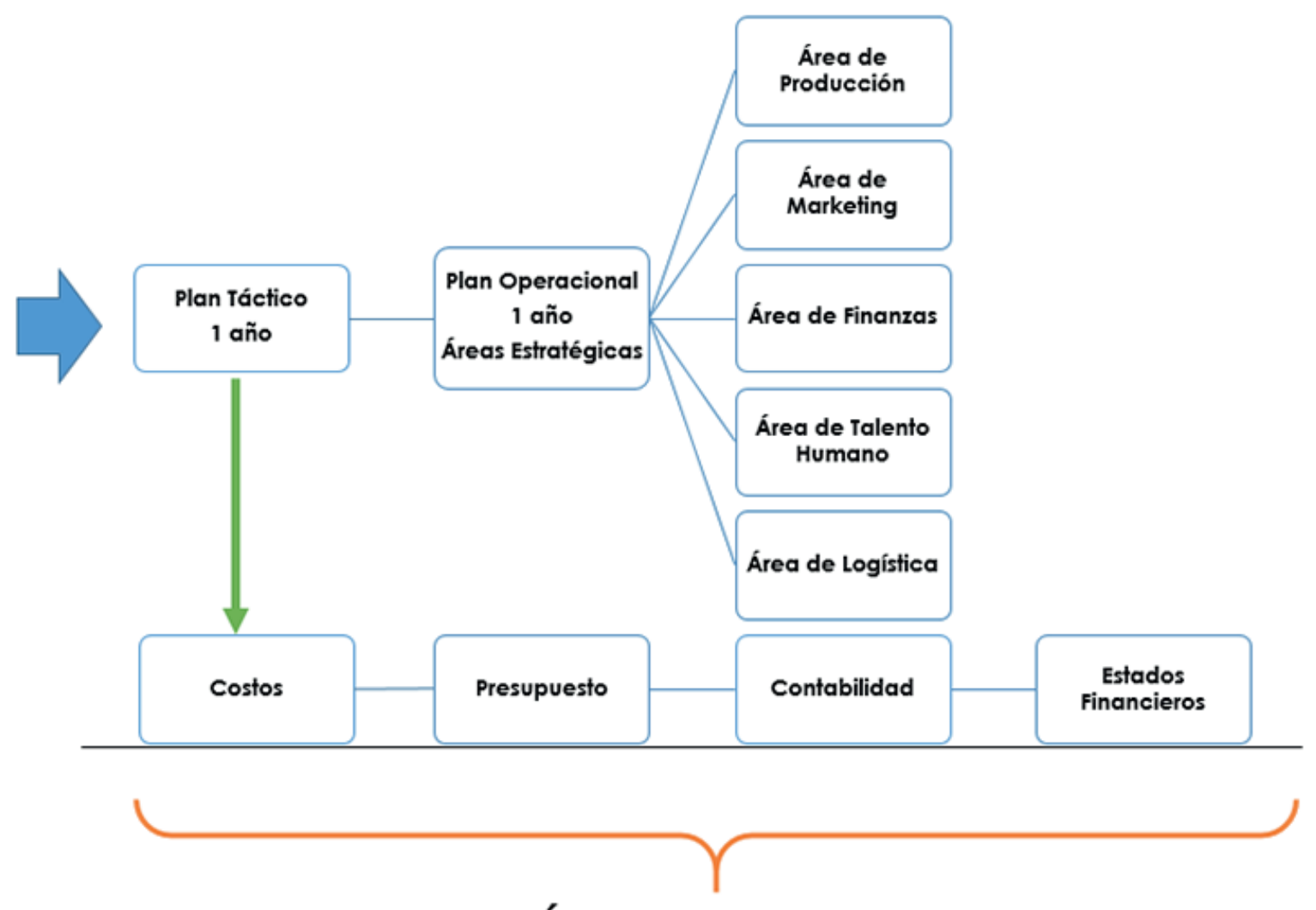

Ámbito Empresarial

Figura 4. Sistema de Planificación del Perú y su Impacto en el Desarrollo Socio-Económico (Parte IV).

Elaboración propia

la iniciativa privada es libre. Se ejerce en una economía social de mercado. Bajo este régimen, el Estado orienta el desarrollo del país, y actúa principalmente en las áreas de promoción del empleo, salud, educación, seguridad, servicios públicos e infraestructura.

Después 26 años de aplicar el mismo modelo económico se aprecia un avance significativo de la economía peruana con sus altas y bajas, motivadas por la crisis mundial y fundamentalmente por las políticas macro económicas aplicadas por los diferentes gobiernos que no tuvieron el resultado esperado y además por la corrupción de la clase política.

Permitió disminuir a la mitad nuestra pobreza e incorporar a más peruanos a la clase media entre 1993 y el 2018, el PBI per cápita real, medido en soles constantes del 2007, creció a una tasa promedio anual de 3,5\%.

La inversión genera valor en el crecimiento de una economía y empleo. La inversión pública y privada no tuvo el impacto esperado, la inversión privada pasó de representar el 12,9\% del PBI en 1993 a 16,7\% en el 2018 y la inversión pública fue de 5,9\% del PBI.

Para asegurar el crecimiento sostenido del país se requiere que el modelo económico se sustente en una planificación nacional, en la que el sector público y privado deben trabajar estrechamente, teniendo como premisas:

- Mejorar el nivel de vida e impulsar el crecimiento económico inclusivo

- Desarrollar infraestructura con crecimiento económico y social

- Adquirir nuevas herramientas técnicas para apostar por la innovación en las organizaciones

- Crear Parques Científicos Tecnológicos como Instrumento de Innovación y Competitividad.

- Apostar por la diversificación productiva. 
- Potenciar la Responsabilidad Social convirtiéndola en protagonista de la planificación nacional.

\section{REFERENCIAS BIBLIOGRAFICAS}

Centro Nacional de Planeamiento Estratégico (2011). CEPLAN. Plan Bicentenario: el Perú hacia el 2021. Recuperado de https://www. ceplan.gob.pe/documentos_/plan-bicentenario-el-peru-hacia-el-2021/

Fondo Monetario Internacional. (2018). Perspectivas Económicas: Las Américas - Una recuperación despareja; octubre de 2018.

Instituto Nacional de Estadística e Informática (2017). INEI: Evolución de la Pobreza Mone- taria 2007-2016. (2017, mayo). Recuperado de https://www.inei.gob.pe/media/MenuRecursivo/publicaciones_digitales/Est/ Lib1425/

Lavigne, M. (2013). Sistemas de protección social en América Latina y el Caribe: Perú. Recuperado de https://repositorio.cepal.org/bitstream/handle/11362/4047/1/S2013058_es.pdf

PISA 2018. (s. f.). Recuperado 28 de octubre de 2019, de http://umc.minedu.gob.pe/pisa-2018/

Valeriano, L. (2018). Planeamiento Estratégico Empresarial. Lo que todo Administrador debe saber al aplicar las Matrices y el BSC (Primera). Lima: San Marcos. 